\title{
TUMOR ESTROMAL GASTROINTESTINAL: ESTUDO DE OITO CASOS
}

\author{
Enio Campos Amico*, Cesar Wilson Bastos Coimbra, luiz Antônio Bugalho, Gisele Alborghetti Nal, Ricardo Marcondes Matos, Cibele Chuery
} Machado, Fabrício Colacino Silva

Trabalho realizado no Hospital Universitário da Faculdade de Medicina da Universidade do Oeste Paulista, Presidente Prudente, São Paulo, SP.

*orrespondência: Rua Poti Nóbrega, 333, ap. 902, Lagoa Nova, Natal, RN, Cep: 59056-180

\begin{abstract}
RESUMO
OBjetivo. O diagnóstico e tratamento dos tumores estromais gastrointestinais (TEGI) têm evoluído a partir de estudos recentes de genética e biologia molecular. Tais avanç̧os têm refletido em melhor sobrevivência dos doentes.

Métodos. Foi realizado estudo retrospectivo no período de novembro//998 a julho/2004, em instituição universitária e em clínica privada dos autores, que identificou portadores de TEGI a partir de positividade para c-kit (CD II7), ao exame imunoistoquímico.

Resultados. Dos oito pacientes estudados, seis eram do sexo feminino (75\%), a idade média foi de 53,2 anos. A presença de massa abdominal palpável foi a apresentaçãa mais comum (62,5\%). 0 estômago foi o órgão mais acometido (62,5\%) seguido pelo intestino delgado (37,5\%). O tamanho médio do tumor foi de $10,6 \mathrm{~cm}$. Em todos, foi possível a ressecção com margens livres. Em quatro doentes houve recidiva local ou à distância, todos esses foram tratados com mesilato de imatinib, com resposta parcial em três e completa em um deles. Sete pacientes (87,5\%) estão vivos em um período de seguimento médio de 33,4 meses. Não foi possível avaliar o comportamento biológico do tumor a partir das variáveis estudadas em virtude da pequena casuística.

Conclusöes. Com base nos dados obtidos de oito pacientes com TEGI do presente estudo, observamos: prevalência dos TEGI em doentes do sexo feminino o estômago como órgão preferencialmente acometido, ausência de correlação entre tamanho do tumor e número de mitoses à análise histológica e taxa de sobrevivência de 87,5\% com um seguimento médio de 33,4 meses.
\end{abstract}

UnITERMOs: Neoplasias gastrointestinais. Sarcoma. Leiomiossarcoma. Imunoistoquímica. Prognóstico.

\section{INTRODUÇÃO}

Os tumores estromais gastrointestinais (TEGI), embora representem menos de $1 \%$ de todas as neoplasias malignas do trato gastrointestinal, constituem o mais importante grupo de tumores mesenquimais primários do aparelho digestivo. São conhecidos por sua diversidade quanto ao comportamento biológico e dificuldades em se determinar o prognóstico dos acometidos!.

O termo tumor estromal foi utilizado pela primeira vez em 1983 por Mazur e Clark para denominar tumores gástricos que supostamente tinham origem no músculo liso, mas que à imunoistoquímica ou microscopia eletrônica não demonstravam claramente origem muscular ou neural2. Progressivamente, o termo TEGI foi adotado para essa categoria específica de neoplasias mesenquimais, benignas ou malignas, originárias no trato gastrointestinal, com fenotipagem incompleta ou mínima para essas linhagens (muscular e neural).

Nos últimos cinco anos, houve um grande avanço no conhecimento de mecanismos biológicos e genéticos que influenciam o surgimento e crescimento dos TEGI. A aplicação desses no diagnóstico e tratamento dessa neoplasia tem sido convertida atualmente em melhora da sobrevivência, mesmo na doença avançada e metastática.

O presente artigo visa analisar: dados clínicos, patológicos e referentes ao tratamento de um pequeno número de doentes tratados pelos autores, à luz desses novos conhecimentos.

\section{Métodos}

Foi realizado o estudo retrospectivo de portadores de TEGI, tratados no período de novembro//998 a julho/2004 pelos três primeiros autores no Hospital Universitário da Universidade do Oeste Paulista e em suas clínicas privadas. Possíveis casos de pacientes tratados anteriormente ao período do estudo não foram investigados por dificuldade no levantamento dos prontuários.

O critério de inclusão nesta casuística estabelecido pelos autores foi presença de tumor em algum órgão do aparelho digestivo que, em estudo imunoistoquímico, apresentasse positividade para CD I I7 (c-kit). Dessa forma, todos os tumores diagnosticados, no período do estudo, como leiomioma, leiomiosarcoma e Schwanoma foram submetidos à confirmação imunoistoquímica.

Os doentes foram submetidos a tratamento cirúrgico objetivando a ressecção completa do tumor. Órgãos adjacentes acometidos por tumor, caso fossem passíveis de ressecção cirúrgica, também foram incluídos na peça operatória por meio de ressecção em bloco.

Dados de anatomia patológica como tamanho do tumor, número de mitoses por campo e presença de linfonodos comprometidos foram anotados (Tabela I). O método imunoistoquímico foi realizado com recuperação antigênica por superaquecimento em solução tampão de citrato $10 \mathrm{mM}$ ph $=6,0$, utilizando o método LSAB (Labeled Streptavidina-Biotina), com diluição dos anticorpos primários (monoclonais e/ou policlonais) de 1:50 a l:1000. 
O tratamento medicamentoso por meio do mesilato de imatinib (STI 57I) foi oferecido a todos os pacientes com recidiva tumoral pós-operatória.

O seguimento pós-operatório foi realizado de acordo com a rotina dos serviços envolvidos; adicionalmente, à época da escrita do presente estudo, todos os pacientes foram convocados por telefone para nova avaliação clínica complementada por realização de exame de ultra-sonografia ou tomografia de abdome.

\section{Resultados}

De um total de dez pacientes analisados, em oito o diagnóstico de TEGI foi confirmado na imunoistoquímica por serem positivos para c-kit.

Dos casos incluídos, cinco já tinham o diagnóstico inicial de TEGI, em dois o diagnóstico inicial era de leiomioma, enquanto um diagnóstico era de Schwanoma.

Houve predominância do sexo feminino (75\%) na casuística. A idade variou de 51 a 59 anos (média de 53,2 anos).

A apresentação clínica mais comum foi a massa abdominal palpável $(62,5 \%)$, enquanto outros achados como hemorragia digestiva alta (12,5\%), dor e emagrecimento (12,5\%) foram menos comuns. Em um doente ( $12,5 \%)$, o diagnóstico foi considerado achado de endoscopia, pois não eram referidos sintomas.

A localização preferencial do tumor foi o estômago (62,5\%), seguido do intestino delgado (37,5\%). O tamanho do tumor medido pelo maior eixo variou entre 5 a $17 \mathrm{~cm}$, com um valor médio de 10,6 cm.

Em quatro pacientes, houve a suspeita pré-operatoria de TEGI, enquanto nos demais a indicação operatória se deveu a obstrução intestinal (I), úlcera gástrica hemorrágica (I), suspeita de diverticulite aguda (I) e suspeita de leiomioma gástrico (I). Em todos, inicialmente não havia sinais de doença avançada.

O tratamento operatório empregado foi: gastrectomia parcial (três), ressecção em cunha do estômago (dois) e enterectomia (três). Em três pacientes (37,5\%), houve a necessidade de ressecção de órgãos adjacentes: o cólon sigmóide e ovário (um), segmento III do fígado (um) e baço (um). A ressecção foi considerada curativa com margens livres em todos os operados, que evoluíram bem, sem complicações significativas no pós-operatório.

O tamanho médio dos tumores foi de 10,62 cm; a despeito disso, na maioria dos casos havia um número pequeno de mitoses por campo. Como observado na Tabela I, todos os tumores expressavam o marcador CD | 17.

O seguimento pós-operatório foi possível em todos, com uma variação de 17 meses a 68 meses (média de 33,4 meses). Em apenas um deles, que se encontrava assintomático, não foi possível a realização de exame de imagem para estadiamento, em virtude da não aceitação do mesmo; todos os demais foram submetidos à ultra-sonografia ou tomografia de abdome.

Durante o seguimento pós-operatório, metade dos doentes (quatro) apresentaram recidiva da doença: o caso I com metástases hepáticas, o caso VI com recidiva local associada a metastases hepáticas, o caso VII com recidiva local apenas e o caso VIII com recidiva local associado à metástase pulmonar. Os demais encontravam-se livres de doença.

\section{Figura I - Tomografia de abdome de doente (VII) submetido previamente a tratamento cirúrgico onde se observa recidiva da doença}

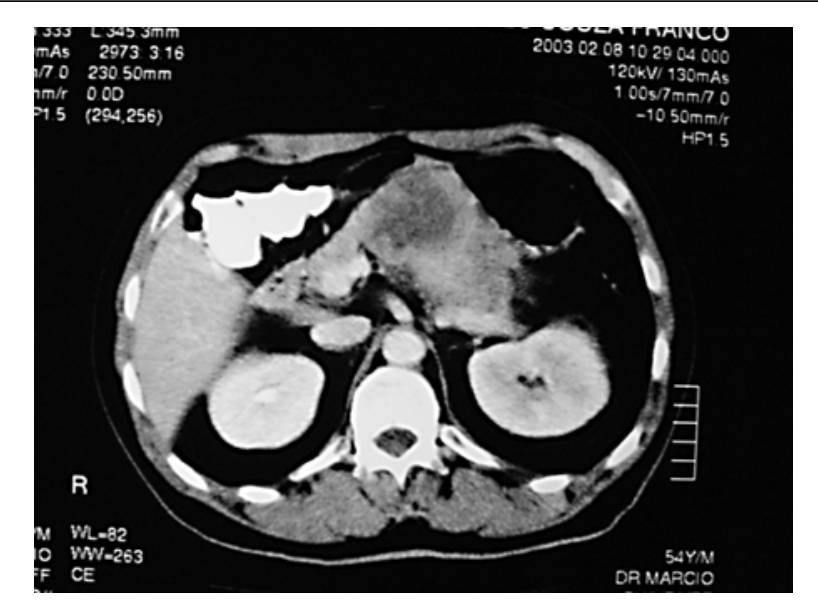

\begin{abstract}
Figura 2 - Tomografia de abdome do mesmo doente, após três meses de tratamento com mesilato de imatinib (caso VII). Nota-se evidente redução da massa tumoral, acompanhada de áreas de necrose tumoral
\end{abstract}

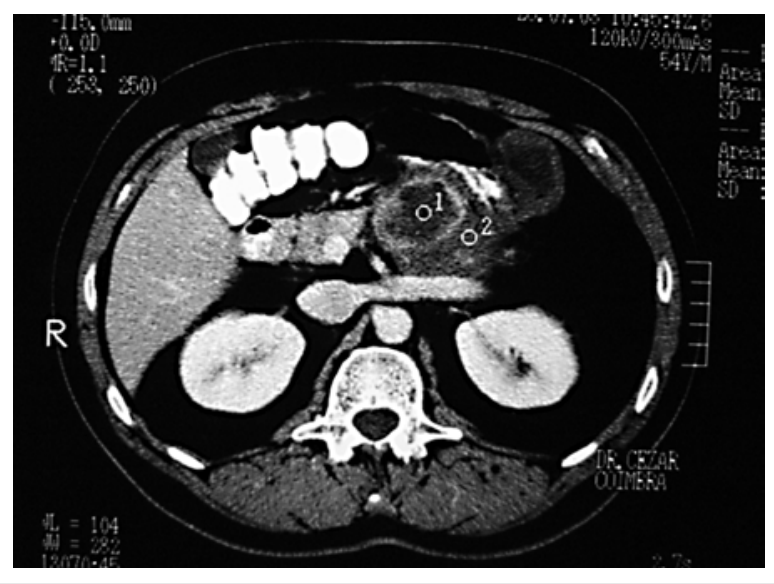

Todos os pacientes em que foi diagnosticada a recidiva da doença foram tratados com o mesilato de imatinib (STI-57I / Gleevec $\circledR$ ) 400 mg via oral em dose única diária, com ótima aceitação e poucos sintomas colaterais. Todos responderam em graus variados a este tratamento. Um paciente (caso VIII) teve resposta completa das metástases pulmonares após 12 meses de tratamento e redução de $90 \%$ da recidiva local (Figs. I e 2), os demais (casos l e VII) apresentaram respostas parciais com redução superior a $50 \%$ do tamanho da massa tumoral com seguimento de 13 e 16 meses (Figura I). Mesmo no único caso de morte da casuística (caso VI), houve uma resposta clínica importante. Dezoito meses após o tratamento operatório e antes do tratamento com o mesilato de imatinib, a paciente encontrava-se caquética, com hepatomegalia e recidiva tumoral palpável em flanco esquerdo. Após dois meses de tratamento, houve desaparecimento da massa e redução do tamanho das metástases hepáticas. A paciente sobreviveu por 24 meses com este tratamento. 
Amico EC ET AL.

Tabela I-Achados macroscópicos, histopatológicos e imunoistoquímicos em oito casos de tumor estromal gastrointestinal

\begin{tabular}{|c|c|c|c|c|c|c|c|}
\hline \multirow[t]{2}{*}{ Doentes } & \multirow{2}{*}{$\begin{array}{c}\text { Tamanho } \\
\text { (Maioreixo/cm) }\end{array}$} & \multirow{2}{*}{$\begin{array}{l}N^{0} \text { de mitoses por } 50 \text { campos } \\
\text { de grande aumento }\end{array}$} & \multirow{2}{*}{$\begin{array}{l}\text { Presença de linfonodos } \\
\text { comprometidos }\end{array}$} & \multicolumn{4}{|c|}{ Imunoistoquímica } \\
\hline & & & & $\overline{C D} \| 17$ & CD 34 & $\$ 100$ & Actina \\
\hline I & 13 & 19 & - & + & + & + & + \\
\hline$\|$ & 8,5 & 3 & - & + & $\mathrm{np}$ & $\mathrm{np}$ & $\mathrm{np}$ \\
\hline$\|||$ & 11,5 & 7 & - & + & $\mathrm{np}$ & $\mathrm{np}$ & $\mathrm{np}$ \\
\hline IV & 8 & 3 & - & + & $\mathrm{np}$ & $\mathrm{np}$ & np \\
\hline V & 5 & 3 & - & t & $\mathrm{np}$ & $\mathrm{np}$ & $\mathrm{np}$ \\
\hline VI & 8 & 3 & - & + & $\mathrm{np}$ & np & np \\
\hline VIII & 17 & 3 & - & + & + & + & + \\
\hline VIII & 14 & 8 & - & + & $\mathrm{np}$ & $\mathrm{np}$ & np \\
\hline
\end{tabular}

\section{Dıscussäo}

Antigamente incluídos no grupo dos leiomiomos ou leiomiosarcomas, sabe-se hoje que os TEGI representam grupos heterogêneos de neoplasia, com aspectos histológicos superpostos, e com evidências de origem não apenas em tecido muscular liso, mas também em bainha de nervo periférico, células neurais do sistema autônomo, células marca-passo (de Cajal) ou mesmo células mesenquimais indiferenciadas ${ }^{3}$.

No final dos anos 90, havia duas correntes dicotômicas para definir os TEGI. A primeira colocava todos os tumores mesenquimais do trato gastrointestinal sob a sigla TEGI. Essa abordagem, embora popular, descaracterizava os leiomiomas e Schwanomas, neoplasias bem definidas do ponto de vista patológico e imunoistoquímico. A segunda abordagem tentava identificar um grupo de tumores mesenquimais que, excluindo os leiomiomas e Schwannomas, apresentavam propriedades histopatológicas comuns. Embora esta última abordagem estivesse mais próxima do pensamento atual, não estava definido um marcador que pudesse identificar esses tumores.

Em meio a essa dificuldade de conceituação, Hirota et al. em 1998 reportaram que alguns TEGI continham uma mutação no exon I I do proto-oncogene, que codifica o receptor tirosina quinase (KIT), expresso em altos níveis nas membranas celulares das células de Cajal ${ }^{4}$. Tal mutação, segundo os autores, permitia um ganho de função do KIT com conseqüente estímulo para o crescimento celular associado a um sinal antiapoptótico descontrolado. Essas alterações favoreceriam o crescimento do clone maligno. A partir disso, a positividade para o CD I 17 (c-kit) à imunoistoquímica tornou-se, com raras exceções, parte do conceito dos TEGI ${ }^{5}$. Em virtude dessa tendência, foram incluídos em nossa casuística aqueles com positividade à imunoistoquímica.

Os TEGI correspondem a $5 \%$ de todos os sarcomas e ocorrem predominantemente em pessoas com média de 60 anos de idade. Quanto à predileção por sexo, a maioria dos autores encontra uma incidência semelhante entre homens e mulheres. Esses dados são diferentes dos encontrados nesta casuística, em que 75\% dos casos pertenciam ao sexo feminino, com uma média de idade de 53,2 anos.

Diferente das pequenas lesões benignas, que são assintomáticas e freqüentemente são achados ocasionais durante exames realizados para outras finalidades, os tumores malignos, por serem maiores, comumente são sintomáticos. As manifestações mais comuns são massa palpável, sangramento digestivo (conseqüente à infiltração mucosa) e dor ${ }^{6}$, o que está de acordo com a sintomatologia dos pacientes desta casuística.

Quanto ao sítio anatômico preferencial, o estômago representa $70 \%$ dos casos, o intestino delgado 20\% a 30\% e menos de 10\% aparecem em qualquer outro lugar do aparelho digestivo ${ }^{5}$. Também aqui, a distribuição do sítio anatômico do tumor em nossos pacientes foi compatível com os achados da literatura, sendo o estômago o órgão mais acometido seguido do intestino delgado, embora a diferença entre as duas incidências em nossa série tenham sido mais discretas $(62,5 \%$ vs $37,5 \%)$.

À macroscopia, os TEGI variam de I até mais de $20 \mathrm{~cm}$, sendo que as lesões maiores podem mostrar degeneração cística, necrose e hemorragia focal. Embora seja incomum, uma pseudocápsula pode ser encontrada ${ }^{7}$. Os tumores ocorrem na submucosa, muscular própria ou serosa e freqüentemente podem atingir a mucosa, o que foi encontrado em um de nossos casos, no qual a endoscopia digestiva sugeriu tratar-se de úlcera gástrica benigna, o que levou a retardo do diagnóstico.

O diagnóstico citológico dos TEGl é freqüentemente suspeito por tais lesões apresentarem disposição celular em fuso (70\%), aspecto epitelióide (20\%) ou ainda aspecto celular misto ${ }^{6}$. 0 primeiro tipo apresenta células com núcleos alongados, bordas cônicas ou arredondadas, com um claro halo perinuclear e citoplasma róseo. O tipo epitelióide apresenta células arredondadas ou com contornos poligonais, com núcleo central ou levemente excêntrico e citoplasma abundante, que pode ser densamente eosinofílico. Qualquer tipo acima descrito pode apresentar aspectos malignos ou benignos, com pleomorfismo nuclear, hipercromasia e nucléolos proeminentes. Os aspectos histológicos dos TEGI por vezes são tão característicos que alguns médicos experientes podem se sentir seguros e fazer o diagnóstico sem a imunoistoquímica ${ }^{5}$. Outros autores, contudo, recomendam este estudo para a confirmação do diagnóstico e para selecionar tratamentos específicos para diferentes pacientes ${ }^{5}$. Para evitar inclusão de casos duvidosos optamos por incluir o paciente nesta série apenas quando a imunoistoquímica fosse positiva para o c-kit. 
Independente do aparecimento recente de novas drogas, o tratamento operatório ainda é considerado o melhor para o TEGl ${ }^{8}$. Todos os tumores, se possível, devem ser ressecados, objetivando sua remoção completa, incluindo os tecidos adjacentes envolvidos. Quando ao exame histopatológico encontram-se margens livres de tumor, considera-se que a ressecção foi curativa. A maioria dos autores não recomenda a ressecção linfonodal extensa como a realizada para o adenocarcinoma, em virtude da pequena incidência de metástase ganglionar 9 . Em nossa série, tivemos um único caso em que houve suspeita deste comprometimento. Após a revisão da lâmina, observou-se que se tratava de implante de TEGI na serosa da grande curvatura gástrica, a poucos centímetros da lesão primária, e não metástase em tecido ganglionar.

Na maior casuística uniinstitucional já publicada, De Matteo et al. relataram a experiência do Memorial Sloan-Kettering no tratamento de 200 TEGls, no período de 1982 a 1998'0. Em II4 pacientes, o tratamento operatório teve intenção curativa, o que só foi confirmado, após o estudo anatomopatológico da peça cirúrgica, em 94 desses pacientes. Para a análise da sobrevivência quando avaliada toda a população de pacientes do estudo, a taxa de sobrevivência livre de doença após cinco anos foi de 35\%. Este resultado foi significativamente melhor (55\%) quando incluídos apenas aqueles com ressecção completa de tumores primários. Em virtude da ineficiência das terapias alternativas, os autores consideravam a reexploração cirúrgica para todos com doença recorrente. Outros autores mostraram resultados semelhantes com o tratamento operatório com índices de sobrevivência variando entre $48 \%$ e $65 \%$ "!

A despeito da ressecção completa com margens livres de neoplasia, a maioria dos tumores recorre ${ }^{6}$ mais comumente na cavidade peritoneal e freqüentemente associada a metástases hepáticas. De importância maior foi o achado que, na maioria dos pacientes, a nova ressecção, a nova ressecção com margens livres, mesmo quando possível, não resultava em maior sobrevida. Esses achados, associados aos desapontadores resultados com quimioterapia convencional e radioterapia, tornavam sombrias, há apenas três anos, as perspectivas para pacientes com recidivas ou doença metastática.

Mudan et al. analisaram seus resultados com 60 pacientes operados após recidiva de TEGI no Memorial Sloan-Kettering Cancer Center no período de 1982 a 1995 e observaram nova recorrência em 76\% dos $\operatorname{casos}^{12}$. De importância maior foi o achado que, na maioria dos pacientes, a nova ressecção com margens livres, mesmo quando possível, não resultava em maior sobrevida. Esses achados, associados aos desapontadores resultados com quimioterapia convencional e radioterapia, tornavam sombrias, há apenas três anos, as perspectivas para pacientes com recidivas ou doença metastática.

O uso do mesilato de imatinib representou uma grande mudança no paradigma do tratamento para o câncer, na qual a terapêutica é dirigida para as anormalidades moleculares específicas na etiologia do tumor $^{13}$. O relato do primeiro paciente portador de TEGI tratado com o mesilato de imatinib foi feito por Joensuu et al., em $200 \mathrm{I}^{14}$. O paciente apresentava uma doença progressiva e metastática, apesar do tratamento com vários esquemas quimioterápicos e inclusive terapia experimental angiogênica. Com poucas semanas de tratamento, o paciente apresentou uma resposta clínica objetiva, mantida por mais de 22 meses. Apenas um ano após, a droga foi aprovada pelo FDA para o tratamento do TEGl ${ }^{7}$. A partir daí, dois estudos clínicos já foram publicados $^{15,16}$. No maior deles, 147 pacientes foram randomizados a receber 400 ou 600 mg de imatinib por dia. A maioria dos pacientes apresentou resposta parcial definida pela redução de pelo menos $50 \%$ do tamanho da lesão (53,7 \% dos casos); a doença foi considerada estabilizada em 27,9\% e apenas 13,6\% apresentaram sua progressão. Não houve diferença no resultado quando se variou a dose empregada ${ }^{16}$. O efeito clínico da droga é rápido, com relatos de melhora dos sintomas após poucos dias de uso. Não é conhecido, no entanto, qual deve ser a duração do tratamento com esta medicação.

O tratamento com o mesilato de imatinib em nossos pacientes foi considerado satisfatório, com resposta parcial na maioria dos casos. A sobrevivência dos pacientes foi de $87,5 \%$, com um seguimento médio de 33,4 meses. Estes achados são superiores aos de De Matteo et al. ${ }^{10}$, na era anterior ao STI-57I, que encontrou sobrevivência de 69\% dos seus pacientes com um ano de seguimento, o que atesta a eficiência da droga a curto e médio prazo. Evidentemente que nada se pode concluir sobre a sobrevida a longo prazo com o uso da droga baseada em nossa pequena casuística.

Quanto ao comportamento biológico do tumor, muitos parâmetros clínicos e histológicos têm sido investigados no sentido de se conhecer suas capacidades preditivas. Três são os mais conhecidos: seu tamanho, o índice mitótico e o sítio do tumor. Dessa forma, os tumores com mais de $5 \mathrm{~cm}$ e mais de cinco mitoses por 50 campos de grande aumento vinham sendo considerados malignos. Da mesma forma, os localizados no intestino delgado teriam prognóstico pior, os de esôfago prognóstico melhor e os demais comportamento intermediário ${ }^{17}$. O que permanece insolúvel quanto à predição do comportamento biológico dos TEGI é o fato de lesões muito pequenas (menores que $2 \mathrm{~cm}$ ) e com baixo índice mitótico (menos de 5 por 50 campos de grande aumento) ocasionalmente metastatizarem ${ }^{5}$. Em virtude disso, um crescente número de centros estão relutantes em usar o termo "benigno" para qualquer TEGI, com exceção de lesões muito pequenas encontradas incidentalmente durante cirurgia abdominal por outras razões ${ }^{5}$. $O$ que parece mais correto na atualidade é considerar a quase totalidade dos TEGI como lesões que apresentam um risco, que pode ser pequeno, intermediário ou grande de comportamento maligno.

\section{Conclusões}

pequeno número de doentes desta série não permite uma conclusão quanto ao comportamento dos tumores, baseados nos parâmetros discutidos acima. No entanto, o caso número III, no qual o tumor media II,5 cm no maior eixo, com sete mitoses em 50 campos de grande aumento, encontrava-se assintomática há cinco anos, enquanto os casos VI e VII, com lesões recidivadas em tempo bem inferior, apresentavam apenas três mitoses por 50 campos de grande aumento. Esses achados sugerem que novos marcadores devam ser investigados a fim de tornar mais previsível o atualmente incerto comportamento biológico dos TEGl18.

Com base nos dados obtidos de oito pacientes com TEGI do presente estudo, observamos: prevalência dos TEGl em doentes do 
Amico EC ET AL.

sexo feminino, o estômago como órgão preferencialmente acometido, ausência de correlação entre tamanho do tumor e número de mitoses à análise histológica e taxa de sobrevivência de 87,5\% nos doentes tratados com ressecção cirúrgica associada, nas recidivas, ao uso de mesilato de imatinib (STI-57I).

\section{Agradecimento}

Ao dr. Antônio Plácido Pereira, médico patologista, pela realização dos estudos imunoistoquímicos e à dra. Gisele Chuery de Amorim pelo auxilio na editoração do artigo.

\section{Conflito de interesse: não há.}

\section{SUMMARY}

Gastrointestinal Stromal tumor: a Report OF EIGHT CASES

INTRODUCTION. Diagnosis and treatment of Gastrointestinal Stromal Tumor have changed because of recent genetic and molecular biological studies which have a direct impact on longer survival.

METHODS. A retrospective research was carried out from November 1998 to July 2004 at the university and in the private clinics of the authors who identified GIST cases based upon positive tests to c-kit (CD / / 7)

RESULTS. The eight patients that were evaluated had an average age of 53.2 and $75 \%$ were females. The most common clinical feature was abdominalmass (62.5\%). The morefrequenttumorsite was the stomach (62.5\%)followed by the small bowel (37.5\%). Mean tumor size was 10.6 $\mathrm{cm}$. Resection with negative microscopic margins was possible in all patients. Tumorrecurrence occurred infour cases which were treated with imatinib mesilate (STI-57I) with partial response in three cases and complete response in the other. Seven patients (87.5\%) are alive with a mean follow-up of 33.4 months ( 9 to 60 months). Due to the limited number of patientsitwas not possible to correlate biological behavior of the tumor with its size and mitotic count.

Conclusions. There was prevalence in the female gender. Most common tumor site was the stomach. Histological examinations did not disclose any correlation between tumor size and number of mitosis. Atan average follow-up of 33.4 months, the mean survival was $87.5 \%$. [Rev Assoc Med Bras 2006; 52(I): 48-52]

KEY wORDS: Gastrointestinal Neoplasms. Sarcoma. Leiomyosarcoma. Immunohistochemistry. Prognosis.

\section{REFERÊNCIAS}

I. Langer C, Gunawan B, Shüler P, Huber W, Füzesi L, Becker H. Prognostic factors influencing surgical management and outcome of gastrointestinal stromal tumours. Br J Surg 2003;90:332-39.

2. Mazur MT, Clark HB. Gastric stromal tumors. Reappraisal of histogenesis. Am J Surg Pathol 1983;7:507-19.
3. Falzoni R. Anatomia patológica dos tumores estromais. In: GamaRodrigues JJ, Lopasso FP, Del Grande JC, Safatle NF, Bresciani C, Malheiros CA, et al. Câncer do estômago. Aspectos atuais do diagnóstico e tratamento. São Paulo: Andrei; 2002. p.313-24.

4. Hirota S, Isozaki K, Moriyama Y, Hashimoto K, Nishida T, Ishiguro S, et al. Gain of function mutations of c-kit in human gastrointestinal stromal tumours. Science 1998;279:577-80.

5. Fletcher CDM, Berman JJ, Corless C, Gorstein F, Lasota J, Longley BJ, et al. Diagnosis of gastrointestinal stromal tumors : a consensus approach. Hum Patol 2002;33:459-65.

6. Pidhorecky I, Cheney RT, Kraybill WG, Gibbs JF. Gastrointestinal stromal tumors : Current diagnosis, biologic behavior, and management. Ann Surg Oncol 2000;7:705-12.

7. Connolly EM, Gaffney E, Reynolds JV. Gatrointestinal stromal tumours. Br J Surg 2003;90: I I78-86.

8. Wu PC, Langerman A, Ryan CW, Hart J, Swiger S, Posner MC. Surgical treatment of gastrointestinal stromal tumors in the imatinib (STI-57 I) era. Surgery 2003; 1 34:656-66.

9. Lev D, Kariv Y, Issakov J, Merhav H, Berger E, Merimsky O, et al. Gastrointestinal stromal sarcomas. Br J Surg 1999;86:545-9.

10. Dematteo RP, Lewis J), Leung D, Mudan SS, Woodruff JM, Brennan MF. Two hundred gastrointestinal stromal tumors. Recurrence patterns and prognostic factors for survival. Ann Surg 2000;23:5I-8.

I I. Ng EH, Pollock RE, Munsell MF, Atkinson EN, Romsdahl JS MM. Prognostic factors influencing survival in gastrointestinal leiomyosarcomas. Implications for surgical management and staging. Ann Surg 1992;21 5:68-77.

12. Mudan SS, Conlon KC, Woodruff JM, Lewis JJ, Brennan MF. Salvage surgery for patients with recurrent gastrointestinal sarcoma : prognostic factors to guide patient selection. Cancer 2000;88:66-74.

13. Dematteo RP, Heinrich MC, El-rifai WM, Demetri G. Clinical management os gastrointestinal stromal tumors : Before and after STI57I. Hum Patol 2002;33:466-77.

14. Joensuu H, Roberts PJ, Sarlomo-Rikala M, Anderson LC, Tervahartiala P, Tuveson D, et al. Effect of the tyrosine kinase inhibitor STI 57I in a patient with a metastatic gastrointestinal stromal tumor. N Engl J Med 200।;344: 1052-56.

I5. Van Oosterom AT, Judson I, Verweij J, Stroobants S, Donato di Paola E, Dimitrijevic S, et al. Safety and efficacy of imatinib (STI57 I) in metastatic gastrointestinal stromal tumours : a phase I study. Lancet 200 I;358: | 42|-3.

16. Demetri GD, Von Mehren M, Blanke CD, Van Den Abbeele AD, Eisenberg B, Roberts PJ, et al. Efficacy and safety of imatinib mesylate in advanced gastrointestinal stromal tumors. $N$ Engl J Med 2002;347:472-80

17. Emory TS, Sobin LH, Lukes L, Lee DH, O'leahy TJ. Prognosis of gastrointestinal smoth muscle (stromal) tumors: dependence on anatomic site. Am J Surg Pathol 1999;23:82-7.

18. Miettinen M, El-Rifai WM, Sobin LH, Lasota J. Evaluation of malignancy and prognosis of gastrointestinal stromal tumors : a review. Hum Patol 2002;33:478-83. 